\title{
Functional Analysis of Pid3-A4, an Ortholog of Rice Blast Resistance Gene Pid3 Revealed by Allele Mining in Common Wild Rice
}

\author{
Qiming Lv, Xiao Xu, Junjun Shang, Guanghuai Jiang, Zhiqian Pang, Zhuangzhi Zhou, Jing Wang, Ya Liu, Ting Li, \\ Xiaobing Li, Jichen Xu, Zhukuan Cheng, Xianfeng Zhao, Shigui Li, and Lihuang Zhu
}

First, eighth, ninth, and fourteenth authors: Rice Research Institute, Sichuan Agricultural University, Chengdu 611130, China; first, second, third, fourth, fifth, sixth, seventh, tenth, twelfth, and thirteenth authors: State Key Laboratory of Plant Genomics and National Plant Gene Research Center (Beijing), Institute of Genetics and Developmental Biology, Chinese Academy of Sciences, Beijing 100101; second and eleventh authors: National Engineering Laboratory for Tree Breeding, Key Laboratory of Genetics and Breeding in Forest Trees and Ornamental Plants, Ministry of Education, The Tree and Ornamental Plant Breeding and Biotechnology Laboratory of State Forestry Administration, Beijing Forestry University, Beijing 100083; and fifth author: Beijing Institute of Genomics, Chinese Academy of Sciences, Beijing 101300.

Accepted for publication 24 January 2013.

\section{ABSTRACT}

Lv, Q., Xu, X., Shang, J., Jiang, G., Pang, Z., Zhou, Z., Wang, J., Liu, Y., Li, T., Li, X., Xu, J., Cheng, Z., Zhao, X., Li, S., and Zhu, L. 2013. Functional analysis of Pid3-A4, an ortholog of rice blast resistance gene Pid3 revealed by allele mining in common wild rice. Phytopathology 103:594-599.

The rice blast resistance gene Pid 3 encodes a nucleotide-binding-site leucine-rich repeat (NBS-LRR) protein. This gene was cloned from the rice 'Digu' (indica) by performing a genome-wide comparison of the NBS-LRR gene family between two genome-sequenced varieties, '9311' (indica) and 'Nipponbare' (japonica). In this study, we performed functional analysis of Pid3-A4, an ortholog of Pid3 revealed by allele mining in the common wild rice A4 (Oryza rufipogon). The predicted protein encoded by Pid3-A4 shares $99.03 \%$ sequence identity with Pid3, with only nine amino-acid substitutions. In wild rice plants, Pid3-A4 is constitutively expressed, and its expression is not induced by Magnaporthe oryzae isolate Zhong-10-8-14 infection. Importantly, in transgenic plants, Pid3-A4, as compared with Pid3, displays a distinct resistance spectrum to a set of $M$. oryzae isolates, including those that prevail in the rice fields of Sichuan Province. Therefore, Pid3-A4 should be quite useful for the breeding of rice blast resistance, especially in southwestern China.

Additional keywords: broad-spectrum resistance.
Rice blast, caused by the filamentous ascomycete Magnaporthe oryzae, is one of the most important diseases of rice. This disease has been found to occur in $>85$ countries, causing an annual worldwide rice yield loss of 10 to $30 \%$ (26). The use of plant varieties with resistance $(R)$ genes is, and will continue to be, the most cost-effective and environmentally-friendly way to control this disease (26).

Thus far, $>80$ rice blast $R$ genes have been described at the genetic level (32), 21 of which have been cloned and characterized. With the exception of Pid2 and the recessive pi21, which encode a receptor-like kinase and a proline-rich protein, respectively $(5,10)$, the remaining 19 cloned $R$ genes (including $P i b$, Pi-ta, Pi9, Piz-t, Pi2, Pi36, Pi37, Rbr2, Pik-m, Pi5, Pid3, Pit, Pb1, Pish, Pik-p, Pik, Pia, Pi54, and Pi25) (3,4,6,18,31-33) all belong to the nucleotide-binding site leucine-rich repeat (NBSLRR) family, representing the largest class of $R$ genes found in plants (7), although Pi54 is not a typical NBS-LRR gene (22). Notably, of the 21 cloned rice blast $R$ genes, 12 have been found to reside in five independent loci on four chromosomesPi37/Pish on chromosome 1 (16,27), Pib/Rbr2 on chromosome 2 (31), Piz-t/Pi2/Pi9 (34) on chromosome 6 near Pid3/Pi25 (3), and Pik/Pik-p/Pik-m $(32,33)$ on chromosome 11 -and each set of allelic or closely linked genes shows a high level of amino acid identity. In addition, at the Pik, Pi5, and Pia locus, their respec-

Corresponding authors: S. Li; E-mail address: lishigui@ sicau.edu.cn; and L. Zhu; E-mail address: lhzhu@genetics.ac.cn

http://dx.doi.org/10.1094/PHYTO-10-12-0260-R

(C) 2013 The American Phytopathological Society tive rice blast resistances have been found to require the presence of two adjacent NBS-LRR genes $(13,18,33)$.

With respect to disease resistance, wild rice species are of great importance as genetic resources for rice breeding, and some $R$ genes have been transferred from wild rice to cultivars. For example, $X a 21, X a 23$, and $X a 27$, known as bacterial blight $R$ genes, were integrated into cultivated rice from the wild rice species Oryza longistaminata, $O$. rufipogon, and $O$. minuta, respectively (14). The rice blast $R$ gene $P i 9$, which is highly resistant to $43 M$. oryzae isolates collected from 13 countries, was also initially transferred from the wild rice species O. minuta (19). The AAgenome common wild rice (O. rufipogon) is closely related to cultivated rice, suggesting that this species is an ancestor of cultivated rice $(28,35)$. Indeed, 60 to $80 \%$ of polymorphisms have been lost during domestication, leading to a reduced number of alleles in cultivated rice (15). Thus, the exploration of new broadspectrum resistance alleles or orthologs in wild rice should be feasible.

We previously cloned Pid3 from the indica 'Digu' by performing a genome-wide comparison of paired NBS-LRR genes and their pseudogene alleles between two rice genome-sequenced varieties, '9311' (indica) and 'Nipponbare' (japonica) (24). Pid3 shows complete resistance to a range of $M$. oryzae isolates, thus playing a significant role in both rice breeding and molecular pathology studies. Subsequently, by performing map-based cloning, Chen et al. (3) demonstrated that the known rice blast $R$ gene Pi25 (29) in another indica, 'Gumei', is actually an allele of Pid3, which encodes a putative protein similar to PID3, with the exception of a single nucleotide substitution at position 459. Notably, Pid3 alleles in most japonica varieties were identified as pseudo- 
genes due to the presence of a nonsense mutation; however, this pseudogene mutation did not occur in the indica varieties that were tested, in African cultivated rice varieties, or in common wild rice species (24). This suggests that some Pid3 alleles in these rice resources may also function as rice blast $R$ genes. The common wild rice A4 (A4) is a rice blast-resistant accession collected in Dongxiang County, Jiangxi Province, China (24). Dongxiang wild rice is known for its tolerance to very low temperatures $\left(-12.8^{\circ} \mathrm{C}\right)(28)$. Thus far, a number of its accessions have been tested for rice blast resistance or susceptibility $(2,20)$ and, to our knowledge, no rice blast $R$ gene has been identified in this wild rice. Pid3-A4, the ortholog of Pid3 in A4, was identified using an allele-mining approach, which consists of a full open reading frame with 2,775 nucleotides and lacking introns (23). In the coding sequence, Pid3-A4 is highly identical to Pid3 $(99.50 \%$ identity). The deduced Pid3-A4 product is a 924-residue polypeptide with the same structure as PID3, belonging to the coiled-

TABLE 1. Results of inoculation of Pid3-A4 and Pid3 transgenic plants with 30 Magnaporthe oryzae isolates ${ }^{\mathrm{a}}$

\begin{tabular}{lllll}
\hline Isolate & \multicolumn{1}{c}{$\begin{array}{c}\text { M. } \text { oryzae } \\
\text { isolate }\end{array}$} & 'Taipei309' & $\begin{array}{c}\text { Pid3-A4 } \\
\text { transgenic line }\end{array}$ & $\begin{array}{c}\text { Pid3 } \\
\text { transgenic line }\end{array}$ \\
\hline 1 & $03-10-66-1$ & $\mathrm{~S}$ & $\mathrm{R}$ & $\mathrm{R}$ \\
2 & $07-31-1-2$ & $\mathrm{~S}$ & $\mathrm{R}$ & $\mathrm{R}$ \\
3 & $03-10-76-3$ & $\mathrm{~S}$ & $\mathrm{R}$ & $\mathrm{R}$ \\
4 & $04-8-2-1$ & $\mathrm{~S}$ & $\mathrm{~S}$ & $\mathrm{~S}$ \\
5 & $10-25-1-1$ & $\mathrm{~S}$ & $\mathrm{R}$ & $\mathrm{S}$ \\
6 & $10-32-2-1$ & $\mathrm{~S}$ & $\mathrm{mR}$ & $\mathrm{mR}$ \\
7 & $10-62-3-1$ & $\mathrm{~S}$ & $\mathrm{mS}$ & $\mathrm{S}$ \\
8 & $10-117-17-1$ & $\mathrm{~S}$ & $\mathrm{~S}$ & $\mathrm{~S}$ \\
9 & $03-11-37-1$ & $\mathrm{~S}$ & $\mathrm{R}$ & $\mathrm{S}$ \\
10 & $07-55-1-1$ & $\mathrm{~S}$ & $\mathrm{R}$ & $\mathrm{R}$ \\
11 & $10-47-9-2$ & $\mathrm{~S}$ & $\mathrm{R}$ & $\mathrm{R}$ \\
12 & $10-120-21-2$ & $\mathrm{~S}$ & $\mathrm{R}$ & $\mathrm{S}$ \\
13 & $07-21-1-1$ & $\mathrm{~S}$ & $\mathrm{R}$ & $\mathrm{R}$ \\
14 & $10-62-2-1$ & $\mathrm{~S}$ & $\mathrm{R}$ & $\mathrm{S}$ \\
15 & $07-24-1-1$ & $\mathrm{~S}$ & $\mathrm{mS}$ & $\mathrm{S}$ \\
16 & $10-31-2-1$ & $\mathrm{~S}$ & $\mathrm{~S}$ & $\mathrm{~S}$ \\
17 & $07-26-22$ & $\mathrm{~S}$ & $\mathrm{R}$ & $\mathrm{R}$ \\
18 & $09-87-2-1$ & $\mathrm{~S}$ & $\mathrm{R}$ & $\mathrm{R}$ \\
19 & $91-65-1$ & $\mathrm{~S}$ & $\mathrm{~S}$ & $\mathrm{~S}$ \\
20 & Sichuan26 & $\mathrm{S}$ & $\mathrm{S}$ & $\mathrm{S}$ \\
21 & $\mathrm{Y34}$ & $\mathrm{S}$ & $\mathrm{R}$ & $\mathrm{R}$ \\
22 & $99-26-2$ & $\mathrm{~S}$ & $\mathrm{mR}$ & $\mathrm{mR}$ \\
23 & CH706 & $\mathrm{S}$ & $\mathrm{R}$ & $\mathrm{S}$ \\
24 & Zhong-10-8-14 & $\mathrm{S}$ & $\mathrm{R}$ & $\mathrm{R}$ \\
25 & ZB15 & $\mathrm{S}$ & $\mathrm{S}$ & $\mathrm{S}$ \\
26 & $97-27-2$ & $\mathrm{~S}$ & $\mathrm{R}$ & $\mathrm{R}$ \\
27 & CH45 & $\mathrm{S}$ & $\mathrm{S}$ & $\mathrm{S}$ \\
28 & JS2001-108-1 & $\mathrm{S}$ & $\mathrm{R}$ & $\mathrm{R}$ \\
29 & $99-20-2$ & $\mathrm{~S}$ & $\mathrm{R}$ & $\mathrm{S}$ \\
30 & $99-26-1$ & $\mathrm{~S}$ & $\mathrm{~S}$ & $\mathrm{R}$ \\
\hline
\end{tabular}

${ }^{\mathrm{a}}$ On a 1 to 5 scale, levels 0 and 1 were considered to be resistant (R); levels 2 and 3 represented medium resistance $(\mathrm{mR})$ and medium susceptibility $(\mathrm{mS})$, respectively; and levels 4 and 5 were considered to be susceptible (S). coil NBS-LRR class of R proteins. There are only nine amino-acid substitutions in the peptide sequence between PID3-A4 and PID3.

Because PID3-A4 shows very high sequence identity with PID3, we presumed that Pid3-A4 may also function as rice blast $R$ genes, like Pid3. Here, we report the functional analysis of Pid3A4. Like Pid3, this Pid3 ortholog is a functional race-specific rice blast $R$ gene and displays a distinct broad-spectrum resistance to a set of $M$. oryzae isolates.

\section{MATERIALS AND METHODS}

Plant materials. A4 is an accession line of the common wild rice $O$. rufipogon collected from Dongxiang County in the Jiangxi Province of China. The susceptible recipient $O$. sativa L. subsp. japonica 'Taipei309' (TP309) and the highly resistant $O$. sativa L subsp. indica Digu are routinely maintained varieties. The species were cultivated in an experimental field of the Institute of Genetics and Developmental Biology in Beijing under normal growing conditions.

Fungal inoculation. $M$. oryzae isolates used in this study are listed in Table 1. Isolates 1 to 18 were collected from rice fields in Sichuan Province of China, and were kindly provided by Dr. Yunliang Peng of Sichuan Academy of Agricultural Sciences (1). Isolates 19 to 30 were collected from other regions of China as well as from Japan, and were kindly provided by Dr. Cailin Lei of Institute of Crop Research, Chinese Academy of Agricultural Sciences $(23,24)$. Isolate Zhong-10-8-14 was used for testing the rice blast resistance of Pid3-A4. The other 29 isolates were used to assay the resistance spectra of both Pid3 and Pid3-A4. Fungal spores were collected with distilled water and diluted to a concentration of $2.5 \times 10^{5}$ conidia $\mathrm{ml}^{-1}$. For inoculation, rice seedlings at the tillering stage were injected with 0.1 to $0.2 \mathrm{ml}$ of spore suspension in the field. The disease rating was evaluated 7 days after inoculation, with the susceptible TP309 as a control. The lesion types on rice leaves were observed and scored from level 0 (resistant) to level 5 (susceptible) using a standard reference scale $(5,25)$. In this study, levels 0 and 1 were considered to be resistant; levels 2 and 3 represented medium resistance and medium susceptibility, respectively; and levels 4 and 5 were considered to be susceptible.

Cloning of the Pid3-A4 promoter region. An $\approx 2,100$-bp-long fragment upstream of the predicted start codon of Pid3-A4 was amplified from A4 genomic DNA using the forward primer Ppid3F: CGTGTACCGTCCTTCTGATTTGAA and reverse primer Ppid3R: GGTTGTCTCGTCGGCGTCCTTGAA. Polymerase chain reaction (PCR) amplification was carried out using the following profile: initial DNA denaturation at $95^{\circ} \mathrm{C}$ for $4 \mathrm{~min}$; followed by 30 cycles of denaturation at $95^{\circ} \mathrm{C}$ for $30 \mathrm{~s}$, annealing at $59^{\circ} \mathrm{C}$ for $30 \mathrm{~s}$, and extension at $72^{\circ} \mathrm{C}$ for $2 \mathrm{~min}$; and final extension at $72^{\circ} \mathrm{C}$ for $5 \mathrm{~min}$.

Vector construction and rice transformation. Forward primer Pid3F: 5'-TTTCTAGAATGGCGGAGGGTGTTGTGGG

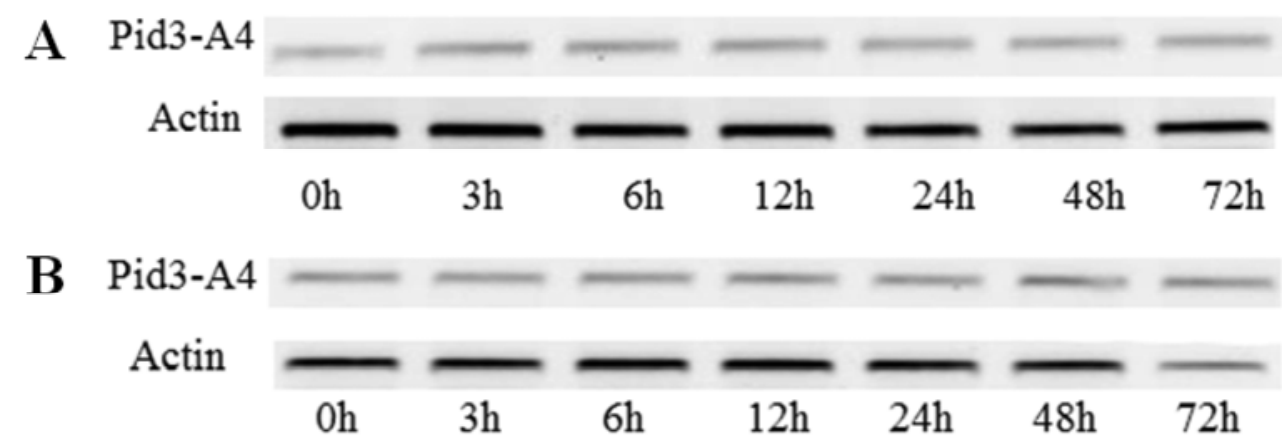

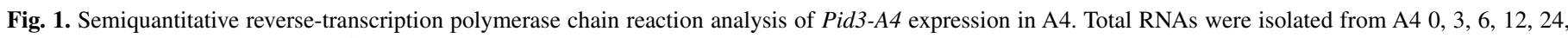
48, and 72 h after inoculation with A, Magnaporthe oryzae Zhong-10-8-14 or B, water. The rice Actin gene was used as a control. 
CTC-3' and reverse primer Pid3R: 5'-CTGTCGACTTATTG AATCCTTTCTGCAGCC- $3^{\prime}$ were used to amplify the full coding sequence of Pid3-A4 from A4 genomic DNA. An XbaI and a SalI recognition site (underlined) with two protecting bases (TT and CT) were added to each $5^{\prime}$ end. PCR amplification was carried out using the following profile: initial DNA denaturation at $95^{\circ} \mathrm{C}$ for $4 \mathrm{~min}$; followed by 30 cycles of denaturation at $95^{\circ} \mathrm{C}$ for $30 \mathrm{~s}$, annealing at $58^{\circ} \mathrm{C}$ for $30 \mathrm{~s}$, and extension at $72^{\circ} \mathrm{C}$ for $3 \mathrm{~min}$; and final extension at $72^{\circ} \mathrm{C}$ for $5 \mathrm{~min}$. The PCR products were purified, sequenced, and inserted into the binary vector pZH01 (30) at the XbaI and SalI cloning sites. The final construct was introduced into Agrobacterium tumefaciens LBA4404. Agrobacterium-mediated transformation was performed using calli derived from mature embryos of susceptible rice TP309 according to Hiei et al. (11). Positive transformants were verified by amplification of the marker gene HPT using forward primer HYGF: 5'GACGGTGTCGTCCATCACAGTTT-3' and reverse primer

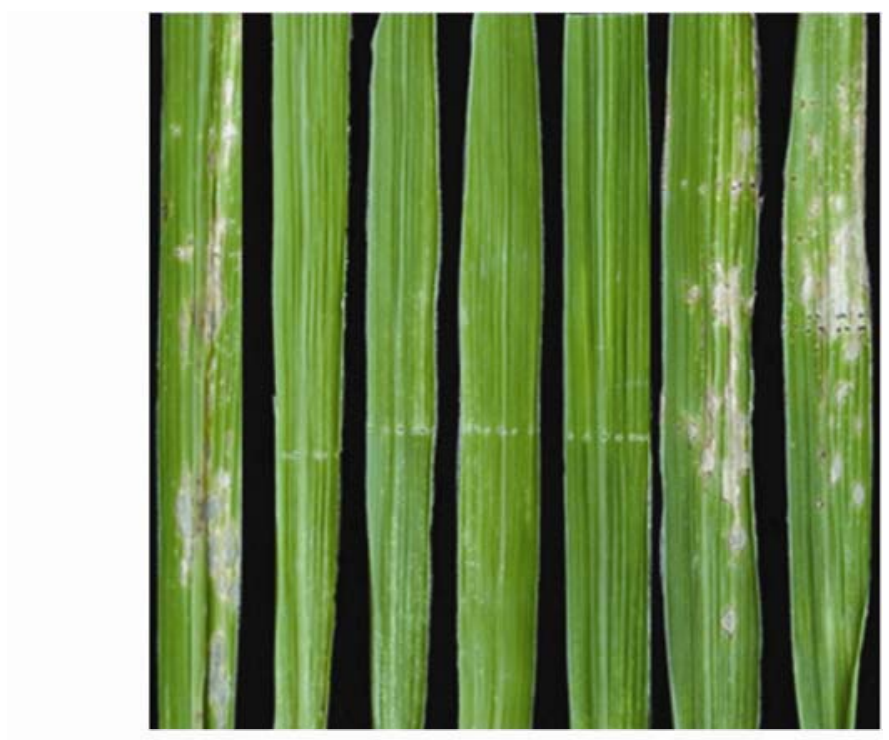

\section{$\operatorname{Pid} 3 \mathrm{C}$}

Actin
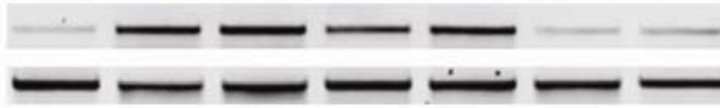

TP309 line1 line2 line3 line4 line 7 line9

Fig. 2. Expression analysis of Pid3-A4 in primary transgenic plants. The recipient 'TP309' and the primary transgenic lines were inoculated with Magnaporthe oryzae Zhong-10-8-14. Expression levels were analyzed using Pid3specific primers Pid3C, which could amplify Pid3-A4 and Pid3-TP309 at the same time. The Actin gene was used as a control.
HYGR: 5'-ACTCACCGCGACGTCTGTCGAGAA-3'. PCR amplification was carried out using the following profile: initial DNA denaturation at $95^{\circ} \mathrm{C}$ for $4 \mathrm{~min}$; followed by 35 cycles of denaturation at $95^{\circ} \mathrm{C}$ for $30 \mathrm{~s}$, annealing at $58^{\circ} \mathrm{C}$ for $30 \mathrm{~s}$, and extension at $72^{\circ} \mathrm{C}$ for $30 \mathrm{~s}$; and final extension at $72^{\circ} \mathrm{C}$ for $5 \mathrm{~min}$. In addition, to confirm positive transformants, the cleaved amplified polymorphic sequence (CAPS) marker Pid3C was designed. This sequence can distinguish Pid3-A4 from Pid3-TP309, based on the nonsense mutation of Pid3-TP309. With the CAPS marker, a 658-bp fragment was amplified using the primer pair $5^{\prime}$ TACTACTCATGGAAGCTAGTTCTC-3' and 5'-ACGTCACAA ATCATTCGCTC-3', followed by digestion with the restriction endoenzyme BamHI. The digested PCR products were resolved on $2 \%$ agarose gels. The presence or absence of a 506-bp restriction fragment was considered to represent positive or negative transformants, respectively.

Expression analysis of Pid3-A4. The resistant A4 and the susceptible TP309 at the tillering stage were inoculated by injection with fungal spores of $M$. oryzae isolate Zhong-10-8-14; water injections were used as control checks. Total RNAs were isolated from leaf sheath tissue with the TRIzol reagent (Invitrogen, Carlsbad, CA) at $0,3,6,12,24,48$, and $72 \mathrm{~h}$. cDNA was synthesized from poly $(\mathrm{A})^{+}$RNA using a cDNA synthesis kit (Transgen, Beijing). Semiquantitative reverse-transcription (RT)-PCR was performed with the Pid3-A4-specific primer pair (Pid3C-F: $5^{\prime}$ TACTACTCATGGAAGCTAGTTCTC-3' and Pid3C-R: 5'-ACGT CACAAATCATTCGCTC-3') for 33 cycles of amplification. Transcription of the Actin gene was used to normalize the cDNA levels with the primer pair 5'-CCTCGTCTCGACCTTGCTGGG$3^{\prime}$ and 5'-GAGAACAAGCAGGAGGACGGC-3'. Amplification of the Actin gene was conducted for 27 cycles. RT-PCR was initiated with one cycle at $95^{\circ} \mathrm{C}$ for 2 min followed by 33 or 27 cycles at $95^{\circ} \mathrm{C}$ for $30 \mathrm{~s}, 58^{\circ} \mathrm{C}$ for $30 \mathrm{~s}$, and $72^{\circ} \mathrm{C}$ for $45 \mathrm{~s}$, and the reaction was terminated with a final extension at $72^{\circ} \mathrm{C}$ for $5 \mathrm{~min}$. The PCR products were resolved on $1 \%$ agarose gels.

The quantitative (q)RT-PCR method was performed to detect the expression patterns of Pid3-A4 or Pid3 in A4, Digu, TP309, and homozygous plants carrying Pid3-A4 or Pid3 driven by the Cauliflower mosaic virus (CaMV) $35 \mathrm{~S}$ promoter. Total RNAs were isolated with the TRIzol reagent (Invitrogen) from leaves. The qRT-PCR analysis was conducted using TransStart Green qPCR SuperMix (Transgen). Primers specific to Pid3-A4 and Pid3 were designed using the GenScript Real-time PCR Primer Design program (https://www.genscript.com/ssl-bin/app/primer) (5'-GAATGCAAATGTTTGGTTCG-3' and 5'-CGCCACATCA TAATTCCTTG-3'); Actin-specific primers (5'-AGCAACTGGGA TGATATGGA-3' and 5'-CAGGGCGATGTAGGAAAGC-3') were used as a standard. The data were normalized to the rice Actin

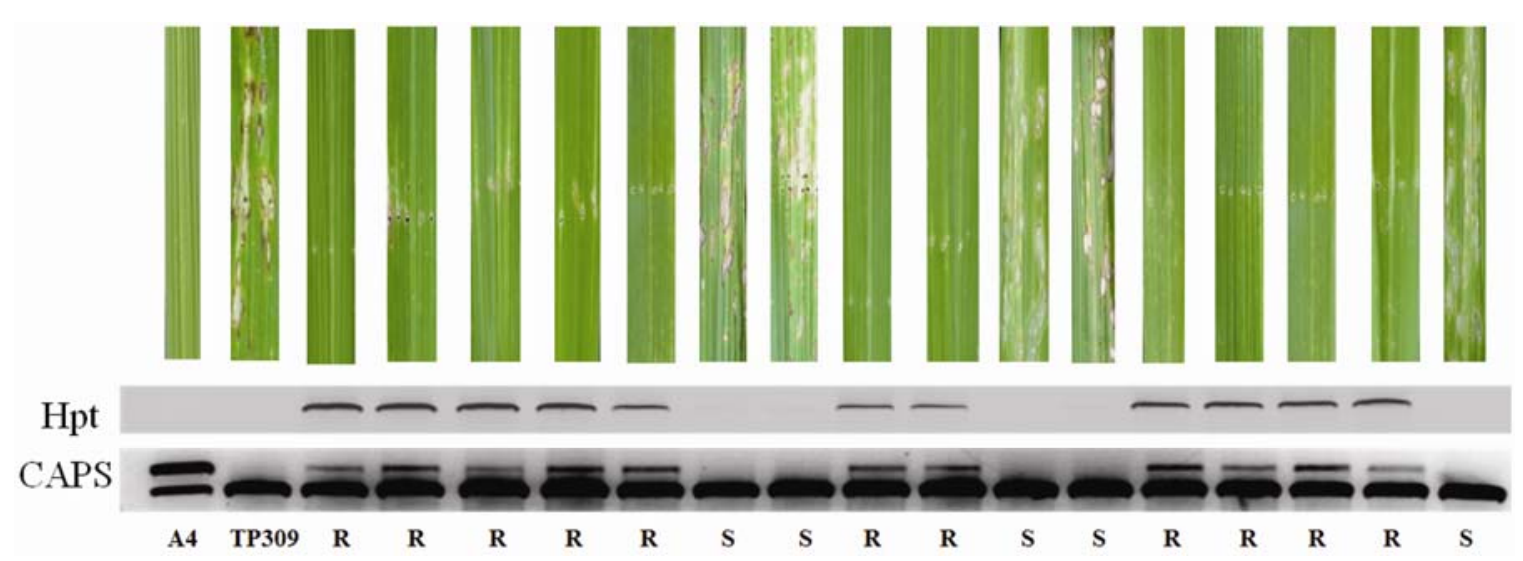

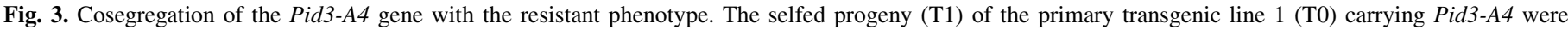

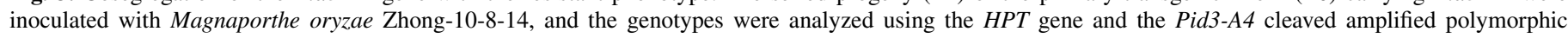
sequence (CAPS) marker. Resistant A4 and susceptible 'TP309' were used as controls. 
gene using the $2^{-\Delta \Delta C T}$ method. The fold change in the expression level was compared with that of sample TP309. The standard error of the mean was calculated. The dissociation curve program was executed to confirm the specificity of the target amplification product.

\section{RESULTS}

Pid3-A4 is constitutively expressed in A4. To compare the predicted promoter region of Pid3-A4 with that of Pid3, we cloned and sequenced an $\approx 2,100$-bp-long fragment (GenBank accession number KC008606) upstream of the predicted start codon of Pid3-A4. The nucleotide identity (96.24\%) in the $5^{\prime}$ upstream regions of the two genes was found to be lower than that in the coding regions. The $5^{\prime}$ upstream region of Pid3-A4 has an 8- and 9-bp deletion, in addition to many single-nucleotide pro-

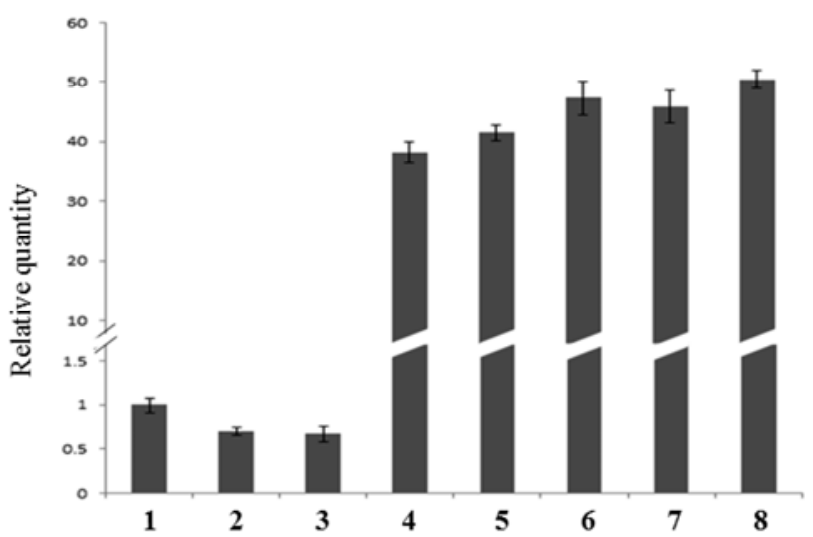

Fig. 4. Quantitative reverse-transcription polymerase chain reaction analysis of the transcript levels of orthologs of Pid3. Relative quantity chart showing the expression levels of orthologs of Pid3 in 'TP309', 'Digu', A4, and transgenic plants with Pid3 or Pid3-A4, both driven by the promoter Cauliflower mosaic virus 35S. Columns: 1, TP309; 2, Digu; 3, A4; 4, transgenic plants with Pid3; and 5 to 8, transgenic plants with Pid3-A4. teins (SNPs), compared with that of Pid3. According to the computer program FGENESH (http://www.softberry.com), the two genes should have predicted promoter regions beginning $900 \mathrm{bp}$ from their respective start codons, where the 9-bp deletion and some SNPs exist. To determine whether these changes in the predicted promoter region of Pid3-A4 would affect the expression profile of this gene, semiquantitative RT-PCR was performed with A4 leaves under normal conditions and with leaves infected with M. oryzae Zhong-10-8-14. Like Pid3 in Digu leaves (23), Pid3A4 was constitutively expressed in A4 leaves, and the expression of this gene was not induced by M. oryzae infection (Fig. 1).

Pid3-A4 is a functional rice blast resistance gene. Because PID3-A4 has an amino acid sequence that is highly identical to that of PID3, with no substitution in conserved motifs of the NBS domain, we presumed that Pid3-A4 may function like Pid3. To verify this assumption, a construct containing the full length of the Pid3-A4 coding sequence under the control of the CaMV 35S promoter was introduced into the susceptible TP309, which was the same recipient used for pCaMV35S::Pid3 in our previous study (24). In total, 21 independent primary transgenic lines (T0) were obtained. Of these, 19 lines were resistant and 2 were susceptible to the $M$. oryzae isolate Zhong-10-8-14, which was also employed as the identification isolate for the Pid3 assay (Fig. 2). Moreover, Pid3-A4 transcripts were detected abundantly in the rice blast-resistant lines but not in the two susceptible lines (Fig. 2 ), indicating that the resistance phenotype is associated with the expression of the transgene. It is noteworthy that Pid3-TP309, the ortholog of Pid3 in TP309, is a constitutively expressed pseudogene (23). In the subsequent inoculation test of the T1 progeny of the resistant transgenic lines, we found that rice blast resistance was strictly linked to the presence of the transgenic selectable marker gene HPT and to the specific CAPS marker for Pid3-A4 as well (Fig. 3). Taken together, these results led us to conclude that $P i d 3-A 4$ is a functional rice blast $R$ gene.

The rice blast resistance spectrum of Pid3-A4 is different from that of Pid3. By continuous selfing, we obtained homozygous Pid3-A4 transgenic plants of the T2 generation, which were all resistant to the isolate Zhong-10-8-14. We then used qRT-PCR
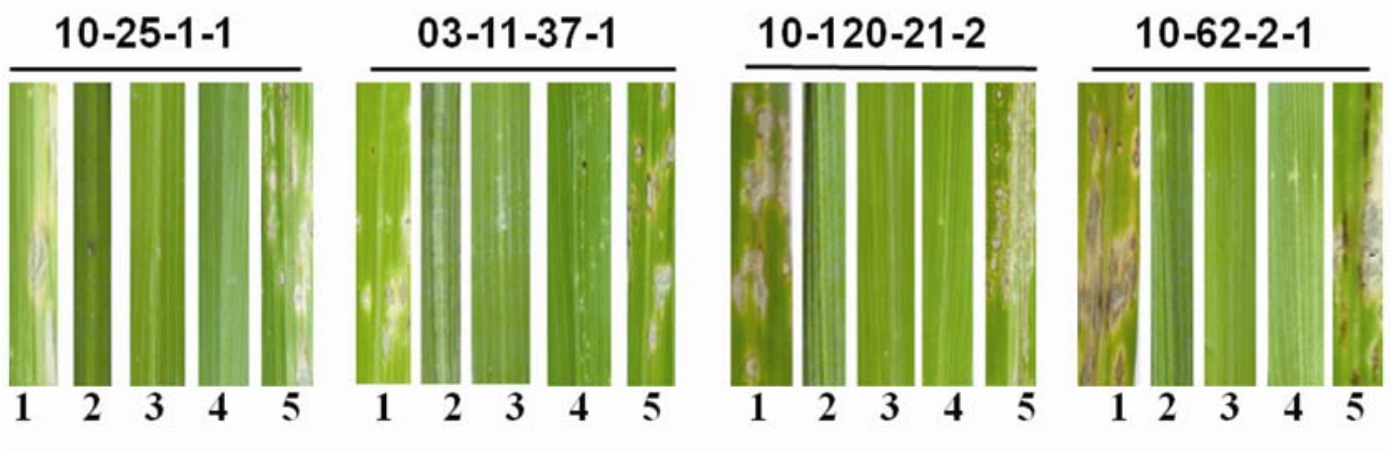

\section{$\mathrm{CH} 706$}

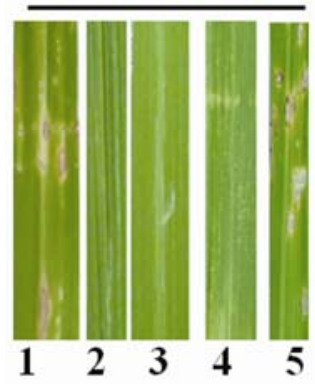

$\mathrm{CH} 45$

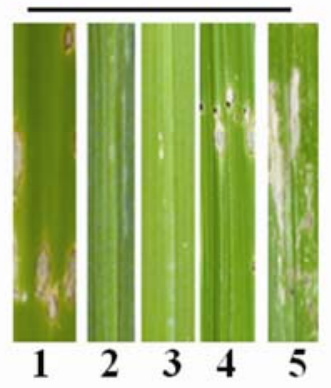

ZB15

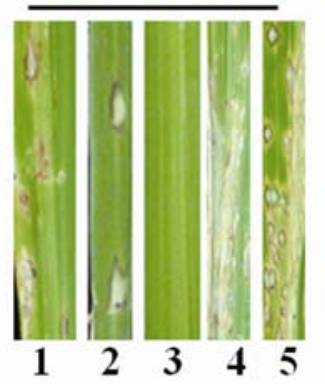

99-26-1

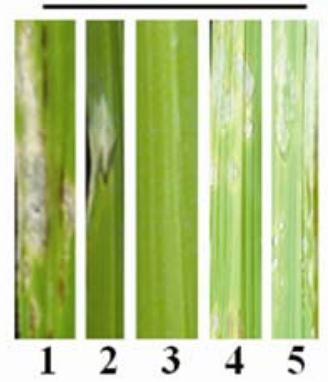

Fig. 5. Phenotyping of rice lines with Magnaporthe oryzae isolates. Disease reaction of 'TP309', 'Digu', A4, and transgenic plants inoculated with M. oryzae 1025-1-1, 03-11-37-1, 10-120-21-2, 10-62-2-1, CH706, CH45, ZB15, and 99-26-1. Columns: 1, TP309; 2, A4; 3, Digu; 4, transgenic plants with Pid3-A4; and 5, transgenic plants with Pid3. 
to quantify the transcript levels of Pid3-TP309, Pid3, and Pid3A4 in the leaves of TP309, Digu, and A4, respectively, and those of pCaMV35S::Pid3 and pCaMV35S::Pid3-A4 in transgenic TP309 plants (Fig. 4). We found that the expression levels of pCaMV35S::Pid3 and pCaMV35S::Pid3-A4 in their respective transgenic plants were approximately the same, although both levels were 40- to 50-fold higher than those of Pid3 and Pid3-A4 in their respective donor plants. The enhanced expression of the two transgenes could be attributed to the effects of the strong promoter CaMV 35S.

To determine whether Pid3-A4 has the same rice blast resistance spectrum as Pid3, we compared the resistance spectra of the two transgenes, both of which showed similar expression levels in the same TP309 background. We used 30 M. oryzae isolates, 18 of which were isolated from fields suffering severe rice blast infection in recent years in Sichuan Province of China (isolates 1 to 18) and 12 isolates that were collected from other regions of China as well as from Japan (isolates 19 to 30). All isolates were compatible with the control TP309 (Table 1). Pid3-A4 transgenic plants showed complete resistance (i.e., race-specific resistance) to 18 isolates and partial resistance to 4 isolates, whereas those of Pid3 showed complete resistance to 13 isolates and partial resistance to 2 isolates. These results demonstrate that, like Pid3, Pid3-A4 is a race-specific rice blast $R$ gene and has a broader resistance spectrum than Pid3 in the range of the tested isolates. Furthermore, Pid3-A4 seemed to be more versatile in response to the test isolates collected from Sichuan Province of southwestern China (Table 1, isolates 1 to 18 ).

To exclude the possibility that the distinct rice blast resistance spectrum of Pid3-A4 resulted from Pid3-A4 overexpression, we also inoculated A4 plants with eight $M$. oryzae isolates to which Pid3 transgenic plants are susceptible (10-25-1-1, 03-11-37-1, 10120-21-2, 10-62-2-1, CH706, CH45, ZB15, and 99-26-1). Like the Pid3-A4-overexpressing transgenic plants, A4 plants showed complete resistance to six isolates and susceptibility to two other isolates (Fig. 5), suggesting that $R$ gene overexpression does not change the resistance spectrum, as was the case for Pid3 (23). It is worthwhile noting that A4 was resistant to $\mathrm{CH} 45$ while Pid3-A4 transgenic plants were susceptible, suggesting that A4 contains additional rice blast $R$ genes.

\section{DISCUSSION}

Although a number of known rice blast $R$ genes have been widely employed in rice breeding, a single $R$ gene cannot provide long-term resistance to the disease. Protection from a single rice blast $R$ gene often proves short-lived due to the rapid variation in $M$. oryzae isolate populations in the field (26). Therefore, it is necessary to identify more rice blast $R$ genes, especially with broad resistance spectrums. Up to now, most of the rice blast $R$ genes were isolated using the labor-intensive map-based approach. We previously identified Pid3-A4 from A4 using an allele-mining approach; in this study, functional analysis of Pid3-A4 showed that it is a functional rice blast $R$ gene and has a broader resistance spectrum than Pid3 in the range of the tested M. oryzae isolates. In addition, recently, the novel rice blast $R$ gene Pi54rh (an ortholog of Pi54) was isolated from the rice-blast-resistant wild rice species $O$. rhizomatis using the allele-mining approach (6). Thus, we believe that allele mining has great potential for revealing new rice blast $R$ genes and is a cost-effective approach that can be applied to identify NBS-LRR-type $R$ genes in other crop species for the purpose of molecular disease resistance breeding.

Plant NBS-LRR proteins are modular. The activity of these proteins depends on their ability to bind and hydrolyze nucleotides $(7,17)$. The N-termini of many NBS-LRR genes have been implicated in accessory protein binding and signal initiation (9). The NBS domain, responsible for the binding and hydrolysis of nucleotides, may be essential for activating downstream signal transduction (21). It has been generally accepted that the C-terminal LRR domain is the major determinant of resistance specificity. The earliest proof was provided by research on the flax $L$ locus; the chimeric genes $L 6-L 2$ and $L 10-L 2$, both of which encode the LRR of $L 2$ in the C-terminal part, confer $L 2$-resistance specificity. Thus, the difference in resistance specificity between L6 and L11 confirmed the results of amino acid differences between their LRR domains (8). The same situation was also observed for the rice Piz locus, where only eight amino acid changes in the LRR domain differentiated PI2 from PIZ-T (34). However, the LRR domain is probably not the only determinant of resistance specificity. For instance, all 11 amino acid differences between L6 and L7 were shown to be located within the first 208 amino acids of the N-terminus, and the CC domain was believed to be important for PIK/PIK-M-resistance specificity $(12,33)$. Here, we show that only 14 nucleotide substitutions between Pid3-A4 and Pid3 result in a nine-amino-acid difference between their peptide sequences. Most of substitutions between PID3 and PID3-A4 are found to be located within the LRR domain, despite the fact that only $\mathrm{D} 856 \mathrm{H}$ is found in the conserved LxxLxLxx motif. Two substitutions are located in the CC domain, and the R44G substitution is found in the nT sequence motif (19). The I259V substitution in the NBS domain is not located within any conserved motif, and both of the residues, isoleucine and valine, are hydrophobic. Therefore, this substitution probably has no influence on the specificity functions of PID3 and PID3-A4. Therefore, the resistance distinction between PID3 and PID3-A4 might be determined by substitutions in the LRR domain or the $\mathrm{CC}$ domain. Currently, we are producing a number of Pid3 orthologs by point mutation and modular recombination, with the aim of clarifying the regions and amino acid residues responsible for blast-resistance specificity.

\section{ACKNOWLEDGMENTS}

This work was supported by grants from the National Basic Research Program of China (2011CB100706 and 2012CB114005), the National Transgenic Research Project (2008ZX08009-001, 2008ZX08009-003, and 2009ZX08001-011B), the National Science Fund for Distinguished Young Scholars of China (31025017), and the National Natural Science Foundation of China (31160223). Q. Lv and X. Xu contributed equally to this work. We thank Y. Peng (Sichuan Academy of Agricultural Sciences) and C. Lei (Institute of Crop Research, Chinese Academy of Agricultural Sciences) for kindly providing $M$. oryzae isolates and $\mathrm{Y}$. $\mathrm{Xu}$ for his critical reading.

\section{LITERATURE CITED}

1. Bai, Y. L. 2011. Virulence to hybrid rice in Magnaporthe oryzae from Sichuan Province. Master dissertation, Sichuan Agricultural University, Chengdu City, Sichuan Province, China.

2. Chen, D. Z., Chen, T. L., Zou, H. H., Xiao, Y. Q., Pi, Y. H., and Yuan, F. L. 2002. Research and utilization of dongxiang wild rice. Acta Agric. Jiangxi 14:51-58. (In Chinese)

3. Chen, J., Shi, Y. F., Liu, W. Z., Chai, R. Y., Fu, Y. P., Zhuang, J. Y., and Wu, J. L. 2011. A Pid3 allele from rice cultivar Gumei2 confers resistance to Magnaporthe oryzae. J. Genet. Genomics 38:209-216.

4. Chen, X. W., and Ronald, P. C. 2011. Innate immunity in rice. Trends Plant Sci. 16:451-459.

5. Chen, X. W., Shang, J. J., Chen, D. X., Lei, C. L., Zou, Y., Zhai, W. X., Liu, G. Z., Xu, J. C., Ling, Z. Z., Cao, G., Ma, B. T., Wang, Y. P., Zhao, X. F., Li, S. G., and Zhu, L. H. 2006. A B-lectin receptor kinase gene conferring rice blast resistance. Plant J. 46:794-804.

6. Das, A., Soubam, D., Singh, P., Thakur, S., Singh, N., and Sharma, T. 2012. A novel blast resistance gene,Pi54rh cloned from wild species of rice, Oryza rhizomatis confers broad spectrum resistance to Magnaporthe oryzae. Funct. Integr. Genomics 12:215-228.

7. Eitas, T. K., and Dangl, J. L. 2010. NB-LRR proteins: Pairs, pieces, perception, partners, and pathways. Curr. Opin. Plant Biol. 13:472-477.

8. Ellis, J. G., Lawrence, G. J., Luck, J. E., and Dodds, P. N. 1999. Identification of regions in alleles of the flax rust resistance gene $L$ that determine differences in gene-for-gene specificity. Plant Cell 11:495-506. 
9. Elmore, J. M., Lin, Z. J. D., and Coaker, G. 2011. Plant NB-LRR signaling: Upstreams and downstreams. Curr. Opin. Plant Biol. 14:365-371.

10. Fukuoka, S., Saka, N., Koga, H., Ono, K., Shimizu, T., Ebana, K., Hayashi, N., Takahashi, A., Hirochika, H., Okuno, K., and Yano, M. 2009. Loss of function of a proline-containing protein confers durable disease resistance in rice. Science 325:998-1001.

11. Hiei, Y., Ohta, S., Komari, T., and Kumashiro, T. 1994. Efficient transformation of rice (Oryza sativa L.) mediated by Agrobacterium and sequence analysis of the boundaries of the T-DNA. Plant J. 6:271-282.

12. Kanzaki, H., Yoshida, K., Saitoh, H., Fujisaki, K., Hirabuchi, A., Allaux, L., Fournier, E., Tharreau, D., and Terauchi, R. 2012. Arms race coevolution of Magnaporthe oryzae AVR-Pik and rice Pik genes driven by their physical interactions. Plant J. Online publication. doi: 10.1111/j.1365-313X.2012.05110.X

13. Lee, S. K., Song, M. Y., Seo, Y. S., Kim, H. K., Ko, S., Cao, P. J., Suh, J. P., Yi, G., Roh, J. H., Lee, S., An, G., Hahn, T. R., Wang, G. L., Ronald, P., and Jeon, J. S. 2009. Rice Pi5-mediated resistance to Magnaporthe oryzae requires the presence of two coiled-coil-nucleotide-bindingleucine-rich repeat genes. Genetics 181:1627-1638.

14. Li, H. J., Li, X. H., Xiao, J. H., Wing, R. A., and Wang, S. P. 2012. Ortholog alleles at $\mathrm{Xa3} / \mathrm{Xa26}$ locus confer conserved race-specific resistance against Xanthomonas oryzae in rice. Mol. Plant 5:281-290.

15. Li, X. R., Tan, L. B., Zhu, Z. F., Huang, H. Y., Liu, Y., Hu, S. N., and Sun, C. Q. 2009. Patterns of nucleotide diversity in wild and cultivated rice. Plant Syst. Evol. 281:97-106.

16. Lin, F., Chen, S., Que, Z. Q., Wang, L., Liu, X. Q., and Pan, Q. H. 2007. The blast resistance gene Pi37 encodes a nucleotide binding site-leucinerich repeat protein and is a member of a resistance gene cluster on rice chromosome 1. Genetics 177:1871-1880.

17. Lukasik, E., and Takken, F. L. W. 2009. STANDing strong, resistance proteins instigators of plant defence. Curr. Opin. Plant Biol. 12:427-436.

18. Okuyama, Y., Kanzaki, H., Abe, A., Yoshida, K., Tamiru, M., Saitoh, H., Fujibe, T., Matsumura, H., Shenton, M., Galam, D. C., Undan, J., Ito, A., Sone, T., and Terauchi, R. 2011. A multifaceted genomics approach allows the isolation of the rice Pia-blast resistance gene consisting of two adjacent NBS-LRR protein genes. Plant J. 66:467-479.

19. Qu, S. H., Liu, G. F., Zhou, B., Bellizzi, M., Zeng, L. R., Dai, L. Y., Han, B., and Wang, G. L. 2006. The broad-spectrum blast resistance gene Pi9 encodes a nucleotide-binding site-leucine-rich repeat protein and is a member of a multigene family in rice. Genetics 172:1901-1914.

20. Que, Z. Q., Shen, C. X., and Lu, Q. N. 2011. Research progress on resistance of Dongxiang wild rice genomplasm resources. Guizhou Agric. Sci. 39:12-15. (In Chinese)

21. Rafiqi, M., Bernoux, M., Ellis, J. G., and Dodds, P. N. 2009. In the trenches of plant pathogen recognition: role of NB-LRR proteins. Semin. Cell Dev. Biol. 20:1017-1024.

22. Rai, A. K., Kumar, S. P., Gupta, S. K., Gautam, N., Singh, N. K., and Sharma, T. R. 2011. Functional complementation of rice blast resistance gene Pi-k(h)(Pi54) conferring resistance to diverse strains of Magnaporthe oryzae. J. Plant Biochem. Biotechnol. 20:55-65.
23. Shang, J. J. 2007. Analysis of the rice NBS-LRR gene family and isolation of a new rice blast resistance gene, Pid3. Ph.D. dissertation, Institute of Genetics and Developmental Biology Chinese Academy of Sciences, Beijing City, China.

24. Shang, J. J., Tao, Y., Chen, X. W., Zou, Y., Lei, C. L., Wang, J., Li, X. B., Zhao, X. F., Zhang, M. J., Lu, Z. K., Xu, J. C., Cheng, Z. K., Wan, J. M., and Zhu, L. H. 2009. Identification of a new rice blast resistance gene, Pid3, by genomewide comparison of paired nucleotide-binding siteleucine-rich repeat genes and their pseudogene alleles between the two sequenced rice genomes. Genetics 182:1303-1311.

25. Silue, D., Notteghem, J. L., and Tharreau, D. 1992. Evidence of a genefor-gene relationship in the Oryza sativa-Magnaporthe grisea pathosystem. Phytopathology 82:577-580.

26. Skamnioti, P., and Gurr, S. J. 2009. Against the grain: safeguarding rice from rice blast disease. Trends Biotechnol. 27:141-150.

27. Takahashi, A., Hayashi, N., Miyao, A., and Hirochika, H. 2010. Unique features of the rice blast resistance Pish locus revealed by large scale retrotransposon-tagging. BMC Plant Biol. 10:175.

28. Tian, F., Li, D. J., Fu, Q., Zhu, Z. F., Fu, Y. C., Wang, X. K., and Sun, C. Q. 2006. Construction of introgression lines carrying wild rice (Oryza rufipogon Griff.) segments in cultivated rice (Oryza sativa L.) background and characterization of introgressed segments associated with yieldrelated traits. Theor. Appl. Genet. 112:570-580.

29. Wu, J. L., Fan, Y. Y., Li, D. B., Zheng, K. L., Leung, H., and Zhuang, J. Y. 2005. Genetic control of rice blast resistance in the durably resistant cultivar Gumei 2 against multiple isolates. Theor. Appl. Genet. 111:50-56.

30. Xiao, H., Wang, Y., Liu, D. F., Wang, W. M., Li, X. B., Zhao, X. F., Xu, J. C., Zhai, W. X., and Zhu, L. H. 2003. Functional analysis of the rice AP3 homologue OsMADS16 by RNA interference. Plant Mol. Biol. 52:957966.

31. Yang, H., Chu, Z., H , Fu, J., and Wang, S., P 2008. The major blast resistance QTL rbr2 is an allele of Pib gene. Mol. Plant Breed. 6:213219. (In Chinese)

32. Yuan, B., Zhai, C., Wang, W. J., Zeng, X. S., Xu, X. K., Hu, H. Q., Lin, F., Wang, L., and Pan, Q. H. 2011. The Pik-p resistance to Magnaporthe oryzae in rice is mediated by a pair of closely linked CC-NBS-LRR genes. Theor. Appl. Genet. 122:1017-1028.

33. Zhai, C., Lin, F., Dong, Z. Q., He, X. Y., Yuan, B., Zeng, X. S., Wang, L., and Pan, Q. H. 2011. The isolation and characterization of Pik, a rice blast resistance gene which emerged after rice domestication. New Phytol. 189:321-334.

34. Zhou, B., Qu, S. H., Liu, G. F., Dolan, M., Sakai, H., Lu, G. D., Bellizzi, M., and Wang, G. L. 2006. The eight amino-acid differences within three leucine-rich repeats between $\mathrm{Pi} 2$ and $\mathrm{Piz}$ - $t$ resistance proteins determine the resistance specificity to Magnaporthe grisea. Mol. Plant-Microbe Interact. 19:1216-1228.

35. Zhu, Q. H., Zheng, X. M., Luo, J. C., Gaut, B. S., and Ge, S. 2007. Multilocus analysis of nucleotide variation of Oryza sativa and its wild relatives: Severe bottleneck during domestication of rice. Mol. Biol. Evol. 24:875-888 